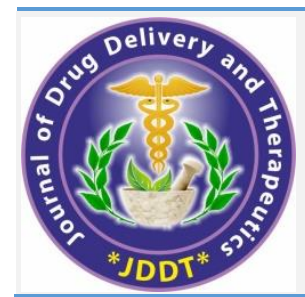

Available online on 15.04.2021 at http://jddtonline.info

Journal of Drug Delivery and Therapeutics

Open Access to Pharmaceutical and Medical Research

(C) 2011-21, publisher and licensee JDDT, This is an Open Access article which permits unrestricted non-commercial use(CC By-NC), provided the original work is properly cited

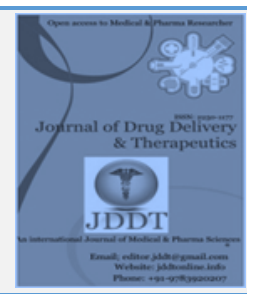

Open Access Full Text Article

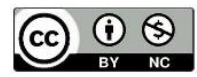

Review Paper

\title{
A Novel Drug Delivery System: Review on Microspheres
}

\author{
Hans Raj, Shagun Sharma, Ankita Sharma, Kapil Kumar Verma, Amit Chaudhary* \\ School of Pharmacy, Abhilashi University, Mandi-175028, Himachal Pradesh, India
}

\begin{tabular}{|c|c|}
\hline \multicolumn{2}{|l|}{ Article Info: } \\
\hline 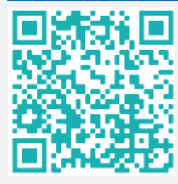 & $\begin{array}{l}\text { Article History: } \\
\text { Received 09 Feb } 2021 \\
\text { Review Completed } 17 \text { March } 2021 \\
\text { Accepted } 23 \text { March } 2021 \\
\text { Available online } 15 \text { April } 2021\end{array}$ \\
\hline \multicolumn{2}{|c|}{ Cite this article as: } \\
\hline \multicolumn{2}{|c|}{$\begin{array}{l}\text { Raj H, Sharma S, Sharma A, Verma KK, Chaudhary A, A } \\
\text { Novel Drug Delivery System: Review on } \\
\text { Microspheres, Journal of Drug Delivery and } \\
\text { Therapeutics. 2021; 11(2-s):156-161 } \\
\text { DOI: http://dx.doi.org/10.22270/jddt.v11i2-s. } 4792\end{array}$} \\
\hline${ }^{*}$ Addr & rrespondence: \\
\hline
\end{tabular}

\section{Abstract}

Microspheres are multiparticulate drug delivery systems that are designed to deliver drugs to a particular location at a fixed rate. Microspheres are free-flowing powders made up of biodegradable proteins or synthetic polymers with particle sizes ranging from 1 to $1000 \mu \mathrm{m}$. Benefits of the use of microspheres in fields such as drug delivery, bone tissue manufacturing, and the absorption and desorption of contaminants by regeneration. The study shows the method of planning and measurement of microsphere parameters. Microspheres are complex, such as bioadhesive microspheres, polymeric microspheres, magnetic microspheres, floating microspheres, radioactive microspheres. Microspheres may be used in various fields such as cosmetics, oral drug delivery, target drug delivery, ophthalmic drug delivery, gene delivery, and others listed in the study. In order to achieve optimal therapeutic effectiveness, it is important to deliver the agent to the target tissue at an optimum level within the right timeframe, resulting in little toxicity and minimal side effects. There are different approaches to supplying the medicinal drug to the target site in a continuous managed manner. One such strategy is the use of microspheres as drug carriers. In this article, the value of the microsphere is seen as a novel drug delivery carrier to achieve site-specific drug delivery was discussed.

Keywords: microspheres, method of preparations, polymer, bioadhesion, types of microspheres

\section{INTRODUCTION}

The new drug delivery system goal is to deliver drugs at a rate that is appropriate to the body's needs during treatment and to get the active ingredient to the site of action as quickly as possible. Drug delivery systems (DDS) with the ability to precisely monitor drug release rates or target drugs to particular body sites have had a profound effect on the health-care system. Over the course of treatment, the best drug delivery device delivers drugs at a set pace determined by the body's needs and delivers the active ingredient to the site of action. By binding the drug to a carrier particle such as microspheres, nanoparticles, or lipids, drug carrier technology offers an intelligent approach to drug delivery. ${ }^{1}$

\section{MICROSPHERES}

Micromatrices are those in which the entrapped substance is dispersed within the microsphere's matrix and microcapsules are those in which the entrapped substance is clearly enclosed by the distinct capsule wall. Micromatrices are those in which the trapped material is dispersed within the microsphere matrix, and microcapsules are those in which the trapped substance is clearly enclosed by a discrete capsule wall. The solid biodegradable microspheres incorporated the drug dispersed or dissolved through the particle- matrix, and they have the potential to allow for regulated drug release. Microspheres are solid spherical particles with diameters ranging from 1 to $1000 \mu \mathrm{m}$. They are biodegradable, spherical, free-flowing particles made up of proteins or synthetic polymers. ${ }^{2}$ There are two types of microspheres;

\section{- Microcapsules}

\section{- Micromatrices}

Microcapsules have a distinct capsule wall around the entrapped material, while micromatrices have the entrapped substance dispersed within the microsphere's matrix. Controlled release of a drug can be accomplished by using solid biodegradable microspheres with a drug dispersed or dissolved via a particle matrix. They are made of biodegradable synthetic polymers and modified natural products, as well as polymeric, waxy, or other protective materials. ${ }^{3}$ They are produced by using polymers and waxes of natural and synthetic materials. The type of polymer used to make microspheres affects their stability, solubility, and drug release. The most popular forms of polymeric microspheres are polyethylene, polystyrene, and expandable microspheres. Microspheres come in both solid and hollow forms. Hollow microspheres are used as additives to reduce a material's density. Microsphere-based topical formulations have gained prominence therapeutic effectiveness for longer periods of time. The use of microparticulate drug delivery systems has become increasingly common in recent years. $4,5,6,7,8$

\section{ADVANTAGES OF MICROSPHERES 9}

$>$ Microspheres have a consistent and long-lasting therapeutic impact. 
$>$ Reduces the frequency of dosing and thereby improves patient compliance.

$>$ Along with their spherical form and smaller size, they may be inserted into the body.

$>$ Improved drug utilization will improve bioavailability while lowering the risk of side effects.

$>$ The morphology of microspheres allows for controlled variability in drug release and degradation.

$>$ Oils and other liquids are converted to solids to make them easier to handle.

\section{CLASSIFICATION OF POLYMER}

Synthetic Polymers: divided into two types:

> Non-biodegradable: Acrolein, Glycidyl methacrylate, Epoxy polymers, etc. 10

> Biodegradable Polyanhydrides, Polyalkylcyanoacryalates Lactides glycosides, and their copolymers. 11,12

Natural materials: They are obtained from different sources like 13,14

$>$ Proteins (albumin, gelatin, collagen)

$>$ Carbohydrate (starch, agarose, carrageenan)

$>$ Chemically modified carbohydrates [poly (acryl dextran), Poly (acryl starch)]

\section{TYPES OF MICROSPHERES}

\section{Bioadhesive microspheres}

Adhesion is the process of attaching a drug to a membrane by using the adhesive properties of water-soluble polymers. Bio adhesion is described as the adhesion of a drug delivery system to a mucosal membrane such as the buccal, ocular, rectal, nasal, and other mucosal membranes. These microspheres have a longer residence period at the application site, resulting in close interaction with the absorption site and improved therapeutic action. ${ }^{15}$

\section{Magnetic microsphere}

This type of delivery system is important because it allows the drug to be delivered to the exact location where it is needed. A smaller amount of magnetically targeted drug will replace a larger amount of freely circulating drug in this condition. Chitosan, dextran, and other integrated materials used in magnetic microspheres have magnetic responses to a magnetic field. ${ }^{16}$ The different types are:

\section{Therapeutic magnetic microspheres}

These are used to administer a chemotherapeutic agent to liver tumors. Drugs like proteins and peptides can also be targeted through this system. ${ }^{15}$

\section{Diagnostic microspheres}

They can be used for imaging liver metastases and also can be used to differentiate bowel loops from other abdominal structures by forming nano-size particles paramagnetic iron oxides. ${ }^{17}$

\section{Floating microspheres}

The bulk density of floating forms is lower than that of gastric fluid, they float in the stomach and do not impact the rate of gastric emptying. If the system is floating on gastric contented, the drug is released slowly and at the desired rate, which increases gastric residence and plasma concentration variability. Strikes and dose dumping are also less likely. It also has a longer therapeutic effect, which decreases dosing frequency. ${ }^{18}$

\section{Radioactive microspheres}

Therapy with Radioembolization Microspheres 10-30 nm in size are larger than capillary microspheres and are tapped in the first capillary bed as they pass through, they are inserted into the arteries that cause a tumour of interest. Thus, under all these cases, radioactive microspheres provide a high dose of radiation to the target areas without affecting normal surrounding tissues. ${ }^{19}$ It differs from the drug delivery system, as radioactivity is not released from microspheres but acts from within a radioisotope typical distance and the different kinds of radioactive microspheres are $\alpha$ emitters, $\beta$ emitters, $\gamma$ emitters. ${ }^{20}$

\section{Polymeric microspheres}

The different types of polymeric microspheres can be classified as:

\section{Biodegradable polymeric microspheres}

Natural polymers like starch are used because they are biodegradable, biocompatible, and bioadhesive. Due to its excellent degree of swelling in an aqueous medium, biodegradable polymer extend the residence time when in contact with mucous membranes, resulting in the formation of gels. The concentration of polymer and the release pattern in a sustained manner regulate the rate and degree of drug release. The main disadvantage is that drug loading performance of biodegradable microspheres in clinical use is complicated, making drug release difficult to manage. However, in microsphere-based therapy, they have a wide variety of applications. ${ }^{21}$

\section{Synthetic polymeric microspheres.}

Synthetic polymeric microspheres are widely used in clinical applications, as well as bulking agents, fillers, embolic particles, drug delivery vehicles, and other applications, and have been shown to be safe and biocompatible. ${ }^{22}$ However, the main downside of these microspheres is that they have a tendency to move away from the injection site, posing a risk of embolism and further organ damage.

\section{MECHANISM OF MICROSPHERES}

The majority of drug delivery via microparticles prevents the formation of a matrix-like internal solid dispersion morphology structure. The drug may be insoluble in the polymeric matrix, and it is released by erosion. First, water diffuses into the matrix, dissolving the resulting near the device's surface. The resulting osmotic pressure is alleviated by forming a channel to the surface and releasing a predetermined amount of drug in the initial drug burst. ${ }^{23}$

\section{METHOD OF PREPRATION}

Methods used for the preparation of microspheres are:

$>$ Single emulsion techniques

$>$ Double emulsion techniques

$>$ Polymerization

- Normal polymerization

- Interfacial polymerization

$>$ Phase separation coacervation technique

$>$ Spray drying

$>$ Emulsion crosslinking method

$>$ Solvent evaporation

$>$ Solution-enhancement dispersion method 


\section{$>$ Ionic gelation method 24}

\section{Single emulsion technique}

This method can be used to prepare a variety of proteins and carbohydrates. The natural polymers are first dissolved in an aqueous medium, then dispersed in an oil phase, which is a non-aqueous medium. That is the initial phase in the process. ${ }^{25}$ Two methods are used to cross-link the next step as:

\section{Cross-linking by heat}

By adding the dispersion into heated oil, but it is unsuitable for the thermolabile drugs.

\section{Chemical cross-linking agents}

By using agents i.e. Formaldehyde, diacid chloride, glutaraldehyde, etc. However, it is detrimental to the undue exposure of active ingredients to chemicals when applied at the time of preparation and then subjected to centrifugation, washing and separation. Chitosan solution (in acetic acid) by applying w/o emulsion to the liquid paraffin containing a surfactant. Microsphere is prepared using a 25 percent solution of glutaraldehyde as a cross-linking agent. 26

\section{Double emulsion technique}

It is the creation of several emulsions, i.e. $\mathrm{W} / \mathrm{O} / \mathrm{W}$ is prepared by pouring the primary w/o emulsion into an aqueous polyvinyl alcohol solution. This w/o/w emulsion shall be put at constant stirring for $30 \mathrm{~min}$. Slowly add some water to the emulsion for a duration of $30 \mathrm{~min}$. Collection of microcapsules by filtration and dry under vacuum. It is ideally suited for water-soluble medicines, peptides, proteins and vaccines. Natural as well as synthetic polymers can be used for this process. The aqueous protein solution is distributed in a continuous organic lipophilic phase. This protein solution will contain active ingredients. ${ }^{27}$ Disperse in oil/organic phase homogenization/vigorous i.e. the formulation of the first emulsion then the addition of the aqueous solution of PVA (Poly Vinyl Alcohol) i.e. the multiple emulsion now produced by the addition of the broad aqueous phase denaturation/hardening after this separation, the washing, drying and collection of the microspheres is prepared using the $\mathrm{o} / \mathrm{w} / \mathrm{o}$ multiple emulsion process. ${ }^{28}$

\section{Polymerization techniques}

Two techniques are mainly used for the formulation of microspheres are as follow;

\section{Normal polymerization}

In bulk polymerization, a monomer or a mixture of a number of monomers along with the initiator or catalyst is usually heated to initiate polymerization. The polymer so obtained may be molded as microspheres. Drug may be done by adding the drug during the process of polymerization. It is a pure polymer formation technique but it is very difficult to dissipate the heat of the reaction which affects the thermolabile the active ingredients. Suspension polymerization is carried out ata lower temperature and also refer to as pearl polymerization in which heating the monomer mixture with the active drug as droplets dispersion in the continuous aqueous phase. ${ }^{29}$

\section{Interfacial polymerization}

The reaction of various monomers at the interface between the two immiscible liquid phases forms a film of polymer that essentially envelops the dispersed phase. In this technique two reacting monomers are employed; one is dissolved in the continuous phase while the other is dispersed in continuous phase (aqueous in nature) throughout which the second monomer is emulsified. ${ }^{29}$

\section{Phase separation coacervation technique}

This method is based on the idea of decreasing the solubility of the polymer in the organic phase in order to influence the formation of a polymer-rich phase called coacervates. In this process, the drug particles are dispersed into a polymer solution and an incompatible polymer is added to the device, which separates the first polymer phase and engulfs the drug particles. Adding the non-solvent results to the solidification of the polymer. This process has been used to prepare polylactic acid (PLA) microspheres by using butadiene as an incompatible polymer. Process variables are very significant as the rate of achievement of the coacervates determines the distribution of the polymer film. The size of the particles and the agglomeration of the formed particles. Agglomeration must be avoided by stirring the suspension using an appropriate speed stirrer, because as the process of microsphere forming starts, the formed polymerized globules begin to adhere and form agglomerates. Process variables are therefore important as they govern the kinetics of the formed particles, since there is no given state of equilibrium attainment. 4,29

\section{Spray Drying}

The polymer is first dissolved in a suitable volatile organic solvent, such as dichloromethane or acetone, before being spray dried. Thereafter, the compound is dispersed in a polymer solution using high-speed homogenization. This dispersion is then atomized in a hot air current. The atomization results in the formation of tiny droplets or fine mist from which the solvent evaporates instantaneously leading to the formation of microspheres in the $1-100 \mu \mathrm{m}$ range. Microparticles are separated from hot air using a cyclone separator, and the solvent residue is removed using vacuum drying. One of the main benefits of the procedure is the viability of action under aseptic conditions. This process is rapid, leading to the formation of porous microparticles. ${ }^{30}$

\section{Emulsion crosslinking method}

This method utilizes the reactive functional group of polymers to crosslink with the aldehyde group of crosslinking agents. In this method water-in-oil (w/o) emulsion was prepared by emulsifying the polymer aqueous solution in the oily phase. Aqueous droplets were stabilized using a suitable surfactant like span 80 or dioctyl sodium sulphosuccinate. The stable emulsion was cross-linked by using an appropriate cross-linker like glutaraldehyde to harden the droplets. Microspheres were filtered and washed repeatedly with hexane or petroleum ether to remove traces of oils. They were eventually washed with water to clear the cross-linkers and then dried at room temperature for 24 hours. ${ }^{31}$

\section{Solvent Evaporation}

Processes are carried out in a liquid production vehicle. The microcapsule is dispersed by a volatile solvent that is not mixed with the liquid stage of the method of production. The core material to be microencapsulated is dissolved or dispersed in a polymer coating solution. With agitation, the core material mixture is distributed during the liquid manufacturing process of the vehicle in order to achieve the required size of the microcapsule. The mixture is then heated, if possible, to evaporate the solvent for the polymer of the main material dispersed in the polymer solution, the polymer shrinks around the core. If the core material is dissolved in a polymer coating solution, a matrix - a form of 
microcapsules is formed. Core materials can be either watersoluble or water-insoluble. Core materials can be watersoluble or water-insoluble. Solvent evaporation entails aqueous $(\mathrm{o} / \mathrm{w})$ or non-aqueous formations. ${ }^{32}$

\section{Ionic gelation method}

This technique was used to prepare the alginate/chitosan particulate system for the release of diclofenac sodium. In this step, the drug is added to an aqueous sodium alginate solution. In order to obtain a complete solution, the stirring continues and the solution containing $\mathrm{Ca} 2+/ \mathrm{Al} 3+$ is added dropwise. The microspheres produced were held in the original solution for 24 hours for internal jellification followed by filtration for separation. The full release is obtained at $\mathrm{pH}$ 6.4-7.2, but the medication will not release at acidic $\mathrm{pH}^{33}$

\section{EVALUATIONPARAMETERS OF MICROSPHERES}

\section{Characterization}

The characterization of the microparticulate carrier is a significant phenomenon that aids in the development of a suitable carrier for the delivery of proteins, drugs, or antigens. The microstructures of these microspheres vary. The release and stability of the carrier are determined by these microstructures. ${ }^{34}$

\section{Particle size and shape}

The most well-known used procedures to visualize microparticles are conventional light microscopy (LM) and scanning electron microscopy (SEM). Both can be used to analyze the shape and outer structure of microparticles. LM provides control over coating parameters in the case of double-walled microspheres. The microspheres structures can be seen before and after coating and the change can be measured microscopically. SEM provides a higher resolution in contrast to the LM. SEM enables the investigation of the surfaces of the microspheres, and when the particles are cross-sectioned, it can also be used for the investigation of double-walled structures. ${ }^{34}$

\section{Electron spectroscopy for chemical analysis}

The surface chemistry of the microspheres can be determined using electron spectroscopy for chemical analysis (ESCA). ${ }^{35}$

\section{Density determination}

The density of the microspheres can be calculated by using a multi-volume pycnometer. ${ }^{36}$

\section{Isoelectric point}

Micro-electrophoresis is used to calculate the electrophoretic mobility of microspheres from which the isoelectric point can be calculated. ${ }^{37}$

\section{Angle of contact}

The contact angle is determined to determine the wetting properties of the microparticle carrier. ${ }^{38}$

\section{In vitro methods}

Release studies for a specific type of microsphere are executed using a different suitable dissolution medium, often by rotating paddle apparatus (USP/BP). ${ }^{39}$

\section{Drug entrapment efficiency}

The entrapment efficiency of the microspheres or the percent entrapment can be determined by holding the microspheres in the buffer solution and allowing lysing. The lysate obtained is filtered or centrifuged and then subjected to monograph requirements for the determination of active constituents. Drug entrapment efficiency can be calculated using the following equation,

$\%$ Entrapment $=$ Actual content $/$ Theoretical content $x$ 100.40

\section{Percentage yield}

It is calculated as the weight of microspheres obtained from each batch divided by the total weight of drug and polymer used to prepare that batch multiplied by 100.40

\section{Swelling index}

The swelling index of the microsphere was determined by using the formula,

Swelling index $=$ (mass of swollen microspheres - mass of drymicrospheres/mass of dried microspheres). ${ }^{39}$

\section{Flow properties 40,41}

Density:

\section{Bulk density}

It is measured by pouring a sample of microspheres of known weight into a measuring cylinder without tapping and measuring its length, and then dividing the weight by the volume.

Bulk density $=$ wt. of microspheres $/$ bulk volume

\section{Tapped density}

It is determined by pouring a sample of microspheres of known weight into a measuring cylinder \& thoroughly tapping it \& measuring its volume, then dividing the weight by the volume.

Tapped density $=$ wt. of the microspheres/volume after tapping

\section{Hausner's ratio}

Hausner's ratio is the ratio of the tapped density to the bulk density of microspheres \& can be used to predict microspheres flow. A Low Hausner's ratio of $<1.2$ indicates a free-flowing microsphere.

$$
\text { Hausner's ratio = bulk density - tapped density }
$$

\section{Angle of repose}

It is defined as the maximum angle to the horizontal that is attainable by a heap of microspheres. The fixed height cone and the fixed base cone are among the methods available for calculating the angle of repose.

$$
\text { Angle of Repose } \boldsymbol{\theta}=\tan -1 \mathrm{~h} / \mathrm{r}
$$

$r=$ the radius of the base of the heap of microsphere

$\mathrm{h}=$ height of the heap of microsphere

Zeta potential: The polyelectrolyte shell is set up by consolidating chitosan of various atomic load into the W2 stage and the subsequent particles are dictated by zeta potential estimation. 42

\section{Attenuated total reflectance Fourier Transform Infrared Spectroscopy}

FT-IR is used to determine the deterioration of the carrier device polymer matrix. The surface of the microspheres shall be examined by calculating the alternative cumulative reflectance (ATR). The ATR-FTIR shall include details on the 
surface composition of the microspheres, depending on the manufacturing processes and conditions. 43,44

\section{In-Vitro Release Study}

Standard IP/BP/USP disintegration mechanical assembly is used to think about the in-vitro discharge profile in the disintegrating media which is the liquid present at the ingestion site according to the monograph, the use of a pivoting bin or an oar form disintegration contraption.

\section{APPLICATIONS OF MICROSPHERES 20,44,45,46}

\section{Microspheres in vaccine delivery}

An ideal vaccine must meet the following criteria: effectiveness, safety, ease of use, and cost.

Biodegradable vaccine delivery systems for vaccines administered through the parenteral route may be able to overcome the limitations of conventional vaccines.

$>$ Improved antigenicity by adjuvant action

$>$ Modulation of antigen release

$>$ Stabilization of antigen.

\section{Targeting using microparticulate carriers}

The idea of site-specific drug delivery, or targeting, is a wellestablished dogma that is gaining a lot of coverage. The drug's therapeutic effectiveness is dependent on its ability to reach and interact with its target receptors. The ability to leave the pool in a repeatable, effective, and precise manner is at the center of drug action, which is mediated by a carrier system.

\section{Monoclonal antibodies mediated microspheres targeting}

Immune microspheres are monoclonal antibodies that are directed at microspheres. This is a technique for achieving targeted targeting to particular sites. Monoclonal antibodies are molecules with a high degree of specificity. Covalent binding allows Mabs to be directly connected to the microspheres. Any of the methods mentioned below can be used to connect Mabs to microspheres.

$>$ Non-specific adsorption and Specific adsorption

$>$ Direct coupling

$>$ Coupling via reagents

\section{Chemoembolisation}

Chemoembolisation is an endovascular therapy that requires selective tumour arterial embolization and simultaneous or subsequent local delivery of a chemotherapeutic agent.

\section{Imaging}

The particle size range of microspheres is a key factor in deciding how radio-labeled microspheres can be used to image specific locations. The particles inserted intravenously outside of the portal vein become entrapped in the lungs' capillary bed. Using labelled human serum albumin microspheres, this phenomenon is exploited for scintigraphic imaging of tumour masses in the lungs.

\section{Topical porous microspheres}

Micro sponges are porous microspheres with a network of interconnected voids ranging in size from 5 to $300 \mu \mathrm{m}$. The topical carries system is made up of microsponges that can entrap a wide variety of active ingredients such as emollients, fragrances, essential oils, and so on.

\section{Medical application}

Microspheres have a broad variety of medical applications, including the long-term release of proteins, hormones, and peptides, and are used in vaccine delivery for diseases such as hepatitis, influenza, pertussis, diphtheria, and many others. Microspheres are the most effective for DNA plasmid and insulin delivery therapies. Microspheres will be used to target leaky tumour vessels passively and tumour cells and antigens actively through intra-arterial/ intravenous application.

\section{Radioactive microspheres applications}

It can be used for liver and spleen tumour radio embolization, as well as arthritis joint radio synovectomy, local radiotherapy, and interactivity care. It is possible to image the liver, spleen, bone marrow, lung, and even the thrombus in a deep vein thrombosis.

\section{Other Applications}

Fluorescent microspheres can be used in membrane-based technology flow cytometry, cell biology, and fluorescent related immunosorbent assays, among other applications. With promising results, yttrium 90 can be used for both primary and pre-transplant control of HCC.

\section{Colonic Drug Delivery}

The polymer has been used for the specific delivery of insulin to the colon e.g. Chitosan.

\section{Vaginal Drug Delivery}

Chitosan, Gelatin, and PLGA are polymers that have had their primary amino groups modified by adding thioglycolic acid. They are often used to treat mycotic infections of the genitourinary tract.

\section{Targeting by Using Micro Particulate Carriers}

The idea of targeting is a well-established dogma that is currently receiving a lot of coverage. The drug's response is determined by its ability to reach and interact with receptors. Typically, pellets are used, which can be made using extrusion / spherization technology, such as microcrystalline cellulose (MCC) and chitosan.

\section{CONCLUSION}

Microspheres are safer for drug delivery than other types of drug delivery systems because they benefit from improved patient enforcement and targeting accuracy. The microspheres drug delivery system is the most common drug delivery system because of its advantages of continuous and controlled-release action, enhanced stability, reduced dose frequency, dissolution rate, and bioavailability. Microsphere drug delivery system is an efficient and secure drug delivery system that can be used in a variety of applications such as precise drug targeting, floating and vaccine delivery, and so on. Methods of preparation and evaluation of prepared microspheres are widely available and effective. Microspheres are used not only to distribute drugs, but also to image tumors, detect biomolecular interactions, and treat cancer. As a result, microspheres will play an increasingly important role in the medical field in the future.

\section{REFERENCES}

1. Chowdary KPR, Yarraguntla SR. Mucoadhesive microsphere for controlled drug delivery. Biological and Pharmaceutical Bulletin 2004; 1717-24.

2. Inada A, Oue T, Yamashita, Yamasaki M, Oshima T and Matsuyam H. Development of highly water-dispersible complexes between coenzyme Q10 and protein hydrolysates. European Journalof 
Pharmaceutical Sciences. 2019; 136.

3. Chaudhari A, Jadhav KR, Kadam VJ. An overview: Microspheres as a nasal drug delivery system. International Journal of Pharmaceutical Sciences Review and Research. 2010 Nov; 5(1).

4. Jain NK. Controlled and Novel drug delivery; CBS Publishers New Delhi, India; 04 Edition.2004; 236-237, 21.

5. Venkatesan P, Manavalan R, Valliappan K. Microencapsulation: a vital technique in novel drug delivery system. Journal of Pharmaceutical Sciences and Research. 2009 Dec 1; 1(4):26-35.

6. Ramteke KH, Jadhav VB, Dhole SN. Microspheres: as carriers used for novel drug delivery system. IOSR Journal of Pharmacy 2012 Jul; 2(4):44-8.

7. Chein YM. Novel Drug Delivery System, second edition, revised \& expanded, Marcel Dekker, Inc, New York 1992

8. Sachan AK, Gupta A, Arora M, Formulation \& characterization of nanostructured lipid carrier (NLC) based gel for topical delivery of etoricoxib, Journal of drug delivery and therapeutics 2016; 6(2):4-13

9. Verma NK, Alam G, Vishwakarma DK, Mishra JN, Khan WU, Singh AP, Roshan A. Recent Advances in Microspheres Technology for Drug Delivery. International Journal of Pharmaceutical Sciences and Nanotechnology. 2015 May 31; 8(2):2799-813.

10. Khar R.K, Vyas, S.P. Targeted and Controlled Drug DeliveryNovel Carrier Systems., 1st edition. CBS Publications and Distributors, New Delhi; 2002; 417- 418.

11. Kreuter J, Nefzger M, Liehl E, Czok R, Voges R. Microspheres - A Novel Approach in Drug Delivery System. Journal of Pharmaceutical sciences.1983; 72:1146.

12. Singh C, Purohit S, Singh M, Pandey BL. Design and evaluation of microspheres: A Review. Journal of drug delivery research. 2013; 2(2):18-27.

13. Midha K, Nagpal M, Arora S. Microspheres: a recent update. International Journal of Recent Scientific Research. 2015; Jul: 5859-67.

14. Sipai AB, Vandanayadav M, Mamatha Y, Prasanth VV. Mucoadhesive Microsphere An overview. American Journal of Pharmaceutical Research. 2012; 2(1):237-58.

15. Liu G, Yang H, Zhou J, Preparation of magnetic microsphere from water-in-oil emulsion stabilized by block copolymer dispersant. Biomacromolecules. 2005; 6:1280-1288.

16. Shanthi N.C, Gupta R, Mahato K.A. Traditional and Emerging Applications of Microspheres: A Review, International Journal of Pharm Tech Research.2010; 2(1):675-681.

17. Najmuddin M, Ahmed A, Shelar S, Patel V, Khan T. Floating Microspheres of Ketoprofen: Formulation and Evaluation, International Journal of Pharmacy and Pharmaceutical sciences.2010; 2(2):83-87.

18. Hafeli U. Physics and Chemistry Basic of Biotechnology. Focus on biotechnology. Review. Radioactive Microspheres for Medical Application. 2002; 7:213-48.

19. Yadav AV, Mote HH. Development of Biodegradable Starch Microspheres for Intranasal Delivery, Indian Journal of pharmaceutical Sciences. 2008; 70(2):170-174.

20. Saralidze K, Leo $H$, Koole, Menno L, Knetsch W. Polymeric Microspheres for Medical Applications, Materials.2010; 3:33573564.

21. Trivedi P, Vermal, GarudN. Preparation and Characterization of Acelofenac Microspheres, Asian Journal of pharmaceutics.2008; 2(2):110-115.

22. Nikam VK, Gudsoorkar VR, Hiremath SN, Dolas RT, Kashid VA Microspheres-A Novel drug delivery system: An overview; International Journal of Pharmaceuticaland chemical sciences. 2012; 1:113-128.

23. Alagusundaram M, Madhu C, Umashankari K, Attuluri B, Lavanya C, Ramakant S. Microspheres: As novel drug delivery system. International Journal of Chem Tech Research. 2009; 1(3):526534.

24. Sinha V R, Singla AK, Wadhawan S, Kaushik R, Kumria R, Bansal $\mathrm{K}$, Dhawan S. Chitosan microspheres as a potential carrier for drugs. International Journal of Pharmaceutics. 2004; 274:1-33.

25. Jayaprakash S, Halith S M, Mohamed Firthouse $P$ U, Kulaturanpillai K, Abhijith, Nagarajan M. Preparation and evaluation of biodegradable microspheres of methotrexate. Asian Journal of Pharmaceutical sciences.2009; 3:26-9.
26. Sinha V R, Bansal K, Kaushik R, Kumria R, Trehan A. Polycaprolactone microspheres and nanospheres. International Journal of Pharmaceutics . 2004; 278 1-23

27. Kumar A, Jha S, Rawal R, Chauhan PS and Maurya SD. Mucoadhesive microspheres for novel drug delivery system: A Review. American Journal of Pharm Tech Research. 2013, 3(4):197- 13.

28. Meena KP, Dangi JS, Samal PK, Namedo KP. Recent advances in microsphere manufacturing technology. International Journal of Pharmacy and Technology. 2011; 3(1):854-855.

29. Kadam NR, Suvarna V. Microsphere: a brief review. Asian Journal of Biomedical and Pharmaceutical Sciences. 2015 Aug 1; $5(47): 13$.

30. Thanoo BC, Sunny MC, Jayakrishanan A. Cross-linkedchitosan microspheres: Preparation and evaluation as a matrix for the controlled release of pharmaceuticals. Journal of Pharmacy and Pharmacology. 1992; 44:283-286

31. Parmar H, Bakliwal S, Gujarathi N, Rane B, Pawar S. Different method of formulation and evaluation of mucoadhesive microsphere. International Journal of Applied Biology and Pharmaceutical Technology. 2010; 1(3):1157-1167.

32. Moy AC, Mathew ST, Mattapan R, Prasanth VV. Microsphere-An Overview. International Journal of Pharmaceutical and Biomedical Sciences. 2011; 2(2):332-338.

33. Alagusundaram M, Chetty MSC, Umashankari K, Badarinath AV, Lavanya C, Ramakant S. Microspheres as a Novel Drug Delivery System- A Review. International Journal of Chem. Tech Research.2009; 1:526-34

34. Singh C, Purohit S, Singh M, Pandey B.L. Design and evaluation of microspheres: A Review, Journal of Drug Development and Research 2013; 2(2):18- 27.

35. Sahil K, Akanksha M, Premjeet S, Bilandi A, Kapoor B. Microsphere: A review. International Journal of Research in Pharmacy and Chemistry 2011; 1(11):84-98.

36. Pavan K. B, Chandiran I.S, Bhavya B, Sindhuri M Microparticulate drug delivery system: A Review. Indian journal of pharmaceutical science \& research. 2011; 1(1):19- 37

37. Dhakar R.C, Maurya S.D, Sagar B.P.S, Bhagat S, Prajapati S.K, Jain C.P. Variables influencing the drug entrapment efficiency of microspheres: A pharmaceutical review. Der Pharmacia Lettre. 2010; 2(5):102-116

38. Gogu P.K. Preparation \& invivo characterization of spray dried Microspheres formulation Encapsulating 4- chlorocurcumin Indian Journal of Pharmaceutical sciences. 2010; 72:346-352.

39. Alagusundaram M, Chetty MS, Umashankari K, Badarinath AV, Lavanya C, Ramkanth S. Microspheres as a novel drug delivery system: A review. International Journal of Chem Tech Research. 2009; 1(3):526-534.

40. Patel NR, Patel DA, Bharadia PD, Pandya V, Modi V. Microsphere as a novel drug deliver. International Journal of Pharmacy \& Life Sciences. 2011; 2(8).

41. Thadanki M, Chadalawada PK, Lavanya P., Umashankar D Formulation and evaluation of sustained release saxagliptin microspheres by ionotropic gelation method. International Journal of Bioassays. 2017; pg. no. 5328-5333.

42. Dupinder K, Saini Seema S: Variable Effecting Drug Entrapment Efficiency of Microspheres: A Review. International Research Journal of Pharmaceutical and Applied Sciences (IRJPAS); 2013; 3(3):24-28.

43. Sailaja A, Anusha K. Review on microspheres as a drug delivery carrier. International Journal of Advances in Pharmaceutics 2017; 06(05):96-102.

44. Tarun V, Gupta J. Pharmaceutical Application of Microspheres: An Approach for the Treatment of Various Diseases; International Journal of Pharmaceutical Sciences and Research, 2017; 8(1):3257- 3259.

45. Sharma N, Purwar N. and Gupta PC. Microspheres as Drug Carriers for Controlled Drug Delivery: A Review. International Journal of Pharmaceutical Sciences and Research 2015 6(11):4579-87

46. Pradeesh TS, Sunny MC, Varma HK, Ramesh P. Preparation of microstructure hydroxyapatite microspheres using oil in water emulsions. Bull Materials Sci. 2005; 28:383-90. 